\title{
Primera piedra.
}

\section{Bases para el Proyecto Arquitectónico}

Foundation stone. Basis for Architectural Design

\section{Resumen}

Autor:

Jaime J. Ferrer Forés jaime.jose.ferrer@upc.edu

Universitat Politècnica de Catalunya (UPC)

España

Recibido: 30 Jun 2018 Aceptado: 14 Dic 2018
- a enseñanza y el aprendizaje del proyecto en un curso inicial constituyen el argumento central de este artículo, que indaga en los fundamentos teóricos sobre los que se asienta la práctica del proyecto arquitectónico. Se formula un marco teórico que compendia un conjunto transmisible de saberes ordenados en torno a la noción de tipos, topos, tectónica, que proceden de la cultura de la propia disciplina arquitectónica y que emanan de las obras arquitectónicas. El programa docente aborda el proceso de proyecto desde la conceptualización de los términos espaciales, formales y constructivos en un esfuerzo reflexivo, un pensamiento teórico que da sentido y coherencia a la forma arquitectónica. A través de la conceptualización de los fundamentos planteada, el curso propone una reflexión sobre la práctica del proyecto arquitectónico, reivindicando el interés de la arquitectura por el conocimiento disciplinar.

Palabras clave: docencia, aprendizaje, proyectos arquitectónicos, tipos, topos, tectónica.

\section{Abstract:}

The teaching and learning of the project in an initial course constitute the central argument of this article that explores the theoretical foundations on which the practice of the architectural project is based. A theoretical framework is formulated that summarizes a transmissible set of knowledge ordered around the notion of typos, topos, tectonics that come from the culture of the architectural discipline itself and that emanate from architectural works. The teaching program addresses the project process from the conceptualization of the spatial, formal and constructive terms in a reflexive effort, a theoretical thought that gives meaning and coherence to the architectural form. Through the conceptualization of the foundations raised, the course proposes a reflection on the practice of the architectural project vindicating the interest of architecture for disciplinary knowledge.

Keywords: teaching, learning, architectural design, typos, topos, tectonics 


\section{Introducción}

Se plantea un programa docente con unas bases estables, disciplinares y propedéuticas para un curso inicial de proyectos, recuperando la tradición sistemática de los estudios y proyectando una profunda reflexión sobre el entendimiento de la arquitectura. Para Livio Vacchini (2009), "proyectar significa abandonarse al placer de construir el pensamiento" (p.57). Se adopta una actitud teórica de la arquitectura y se parte del conocimiento basado en la obra arquitectónica como material de proyecto.

En este proyecto docente se analiza la metodología y la didáctica de un curso inicial de Proyectos Arquitectónicos y se plantea una propuesta para el primer ciclo de los estudios como aprendizaje acumulativo de la arquitectura a través de "la interacción constante de las tres nociones primordiales del Tipos, Topos, Tectónica" (Frampton, 1999, p.13). El análisis de una obra permite profundizar en las exigencias del programa (Tipos), la naturaleza del contexto (Topos), o los requisitos de la construcción y los materiales (Tectónica). Así, este programa aspira a establecer un sustrato teórico sobre el que se asiente la práctica del proyecto arquitectónico y propone un acercamiento disciplinar al proyecto arquitectónico, basado en el conocimiento y en la orientación propedéutica del programa docente. La presentación abunda sobre el qué se pretende enseñar y transmitir en esta fase temprana del aprendizaje de proyectos.

Como decía Mies van der Rohe, no podemos pretender inventar una arquitectura cada lunes (Schulze y Windhorst, 2016, p.433). Frente a la enseñanza del proyecto como acto reflejo, a partir del encadenamiento de diversos proyectos como simulación de la actividad profesional, se propone un curso de proyectos entendido como una profundización en el conocimiento arquitectónico, mediante un curso reflexivo y una enseñanza activa. Se concibe como una primera aproximación, tanto a la obra construida como al proceso de proyecto, que permita dotar al estudiante de los recursos necesarios para "adquirir conciencia sobre lo que está haciendo, pues de otro modo no pasaría de un mero hacer sin más trascendencia ni posibilidad de aprendizaje" (García del Monte, 2017, p.34).

Reivindicando la voluntad de entender la arquitectura, el profesor Carlos Martí Arís (2005) escribe que "las grandes obras de arquitectura, antiguas y modernas, no pueden entenderse sin la existencia, explícita o implícita, de una labor teórica, de un pensamiento activo, que pone constantemente en relación las formas arquitectónicas concretas con las ideas y los conceptos de que aquellas formas se alimentan. Sin esa labor reflexiva que es propia de la teoría, no cabe hablar de conocimiento" (pp.26-27). Esta labor reflexiva centrará las bases pedagógicas del curso, que indaga en la búsqueda paciente y rigurosa de estos principios permanentes, tanto en la obra construida como en el proceso de proyecto, para comprender sus métodos y procedimientos.

\section{Bases pedagógicas}

En el inicio del primero de los Diez libros de Arquitectura, Vitruvio escribe una certera reflexión a los que se dedican a estudiar la arquitectura: "es preciso tener talento y afición al estudio, puesto que ni el talento sin el estudio, ni el estudio sin talento, pueden formar un buen arquitecto" $(1980$, p.6). Los estudiantes de los primeros cursos de proyectos se encuentran en un terreno desconocido y en los primeros cursos se pretende incidir en el marco referencial de cultura arquitectónica y de las obras de los maestros de la Modernidad para aprehender tanto los valores como las técnicas de proyecto y construcción. Se parte así, de la convicción de que la arquitectura se aprende de la propia arquitectura.

La arquitectura posee un contenido disciplinar que puede ser codificado y transmitido. Sin esta convicción no existe la pedagogía. Así, la claridad y elementalidad son los atributos que definen la orientación pedagógica del programa docente del curso propedéutico en el que se recurre a la codificación de las bases, fundamentos, recursos, métodos y principios del proyecto para propiciar su transmisibilidad.

La vocación didáctica del curso propicia la codificación de los elementos del proyecto arquitectónico y su sistematización, y el programa se plantea como un manual destinado al aprendizaje y a la reflexión que permita guiar al estudiante en su formación disciplinar y en su búsqueda personal del proyecto con el empleo específico de las herramientas del proyecto arquitectónico: espaciales, técnicas y de contexto. Ante la dificultad de proponer un método proyectual, la docencia que se propone impartir en el marco propedéutico del curso inicial asume la lenta y paciente búsqueda que sugiere Le Corbusier a través de la interiorización progresiva de una serie de ideas básicas en torno a la arquitectura. La aspiración del curso es proporcionar el marco de reflexión sobre la disciplina arquitectónica donde se desarrolle el ejercicio del proyecto siguiendo el ejemplo del marco referencial de la tradición, recurriendo a la historia de la cultura arquitectónica, a los fundamentos y las bases de la tradición moderna y desarrollar así un acercamiento disciplinar al proyecto desde el rigor y el conocimiento, reconociendo los logros y medios en cada momento histórico. En consecuencia, para lograr este conocimiento profundo de las obras de arquitectura, se plantea como objetivo docente en este curso iniciático, un enfoque holístico capaz de integrar tanto aspectos objetivos como percepciones subjetivas. 
En el curso de los estudios se planifican también una serie de actividades paralelas a la docencia reglada, con el fin de fomentar la inquietud investigadora del estudiante con un aprendizaje fuera del aula. Se trata así de proyectar y ofrecer un aprendizaje complementario más allá del aula para transmitir al estudiante que él mismo es el protagonista y responsable de su propio aprendizaje.

Los viajes han sido siempre uno de los cauces importantes para la transmisión y el conocimiento de las aportaciones en todos los ámbitos de la cultura. El aprendizaje a través del conocimiento directo a algunas de las obras relevantes visitadas supone una mayor trascendencia en el aprendizaje de los estudiantes (Labarta, 2011, p.44). Conscientes de sus valores pedagógicos, este esfuerzo académico y organizativo, que se desarrolla fuera del calendario académico, pretende acercar a los estudiantes al aprendizaje directo de las obras de arquitectura y de las lecciones urbanas. Se pretende así que los estudiantes se acostumbren a sentir que la experiencia arquitectónica y el proyecto se nutre en la continuada atención a las más diversas actividades.

\section{Espacio}

El curso se basa en la idea de transmisibilidad y racionalidad de la arquitectura y los ejercicios propuestos tratan de clarificar los mecanismos, métodos e instrumentos de la disciplina arquitectónica, no sólo como aprendizaje sino con la intención de formar el juicio crítico del estudiante. La vieja máxima, "me lo contaron y lo olvidé, lo vi y lo entendí, lo hice y lo aprendí" de Confucio, ilustra la voluntad de fijar desde el primer enunciado las coordenadas del programa docente: la comprensión del espacio arquitectónico como elemento específico de la arquitectura.

La primera etapa analítica tiene como principal objetivo presentar una serie de ideas básicas en torno a la arquitectura. El interés de esta etapa consiste en profundizar en estos temas y utilizarlos como base crítica de los proyectos. Bruno Zevi (1991) afirma, "tomar posesión del espacio, saberlo ver, constituye la llave de ingreso a la comprensión de los edificios (...) la historia de la arquitectura es, ante todo, la historia de las concepciones espaciales" (pp. 20-31). El primer análisis del curso se dedica al reconocimiento de lo específico en arquitectura: el espacio, el protagonista de la arquitectura. El primer ejercicio aborda la noción espacial del "Tipos" y se analiza desde el punto de vista de la comprensión del espacio una obra de arquitectura en relación a los elementos y las relaciones.

La primera parte de este ejercicio consiste en el análisis gráfico y descriptivo de una obra de arquitectura a través del redibujo y la construcción de una maqueta. Cuando se redibujan estas obras se comprenden mejor. El dibujo es una manera de aprender a ver y, además, como señala Rafael Moneo, el dibujo es la primera construcción de la arquitectura (Cortés y Moneo, 1976).
Esta primera parte del trabajo comprende la construcción de la maqueta del espacio. La construcción de esta maqueta en la formación del arquitecto, en el curso de los estudios, está destinada a celebrar la comprensión del espacio en arquitectura. El arquitecto romano Luigi Moretti elaboró una referencial maqueta analítica de San Pedro de Roma que representa el vacío (Figura 1).
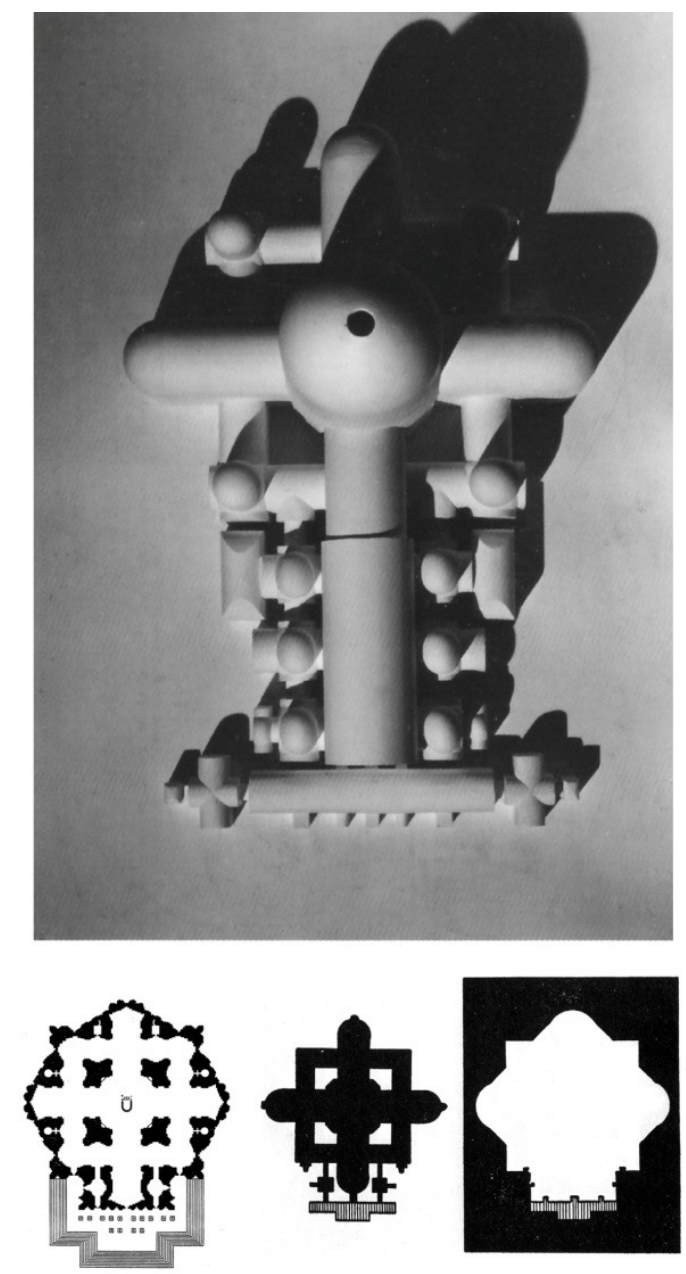

Figura 1: Luigi Moretti. Maqueta del espacio de San Pedro de Roma y análisis de Bruno Zevi del espacio interno y el espacio externo de San Pedro de Roma.

Fuente: Zevi, B. (1991). Saber ver la arquitectura. Barcelona, España: Poseidon, p.20.

La construcción de una maqueta del vacío supone una notable dificultad por el grado de abstracción y el reconocimiento y estudio del volumen vacío. El análisis gráfico que realiza Bruno Zevi del proyecto de San Pedro de Roma de Miguel Ángel (1520) enfatiza la espacialidad interior en contraposición a la envolvente exterior. En la inversión del dibujo de la planta se atribuye al espacio el carácter de figura.

Con la abstracción de la construcción de una maqueta del vacío se pretende insistir en la comprensión del espacio en arquitectura otorgándole el papel de figura, 
de elemento protagonista y no de fondo (Figura 1) Como señala Geoffrey Scott (1970), "delimitar un espacio es la finalidad del construir. Lo que hacemos al proyectar es determinar una cantidad adecuada y conveniente de espacio, cerrarlo y protegerlo" (p.10). Así, el análisis se centra en la forma de la delimitación espacial (composición), la forma corpórea (tratamiento material de los límites) y por último, en la forma visible (tratamiento de la luz).

Una vez analizado el espacio se propone la exposición en él de una obra de arte. Se plantea la experimentación sobre la configuración espacial de un lugar en el que el objetivo sea descubrir y contemplar una obra de arte conocida. Se valora la reflexión sobre la percepción espacial y el recorrido, la abstracción y la especificidad así como la representación y la adecuación del espacio a la obra de arte. Se pretende estudiar cómo el arquitecto recurre al movimiento para enfatizar la percepción de espacio, enriqueciendo la experiencia espacial, mediante la dimensión háptica que se produce al recorrer y percibir un espacio (Alba Dorado, 2016, p.89). El reconocimiento perceptivo de los materiales permitirá al estudiante profundizar en los valores fenomenológicos de la arquitectura que reivindican, entre otros autores, la obra y el pensamiento de Juhani Pallasmaa.

El collage de Mies van der Rohe para una sala de conciertos en el interior de una nave de ensamblaje de bombarderos en Baltimore (1937-1939), obra de Albert Kahn, ilustra la intervención a desarrollar en el espacio estudiado. El collage de Mies van der Rohe de la Casa Resor (1937-1938) o el collage para el proyecto de un Museo para una pequeña ciudad (1942), representan con eficacia la composición definida por Vallespín, Cervero y Cabodevilla-Artieda como "transparencia fenomenal" (2017, p.143)

El ejercicio propone la reflexión en torno a la percepción de una obra de arte en un espacio. En una pintura la percepción se produce estáticamente frente a la obra. La visión de una escultura obliga al observador a moverse en torno al objeto para conseguir una percepción dinámica y cambiante, una visión cinética. En cambio, la envolvente arquitectónica del edificio sitúa en su interior al observador y configura su vivencia espacial.

\section{Tipos}

Se analiza en este apartado el entendimiento de la arquitectura como soporte de la actividad humana. Para el profesor Carlos Martí Arís (2014), "la arquitectura es un procedimiento capaz de dar forma a la actividad" (p.85). En este ámbito se indaga en la interrelación entre programa funcional y forma arquitectónica y se exponen los distintos enfoques de la organización de las actividades que los edificios albergan, de la utilitas vitruviana, desde la concepción funcionalista de "la forma sigue a la función" con el ensamblaje de elementos funcionales de la composición por partes, a la noción de la flexibilidad y la planta libre modernas; desde la idea de organizar el edificio en espacios servidos y espacios servidores que formula Louis Kahn, a la sistemática concepción moderna del mat-building o a la denominada composición aditiva de Jørn Utzon (Capitel, 2016, p.53). La composición es un instrumento para resolver la relación entre forma y programa (Figura 2)

Se aborda así el estudio de las formas recurrentes, de las constantes formales, de los tipos reivindicando la capacidad operativa del principio tipológico que dota al proyecto de una estructura formal consistente. Un tipo es una constante formal de origen histórico que pese al cambio de su uso mantiene el mismo aspecto. Conserva un principio elemental que se puede reconocer y actúa como una eficaz referencia de proyecto. En la tradición clásica, el tipo es un instrumento para la composición. Para el tratadista francés J.N.L. Durand se parte de los elementos (columnas, muros, huecos...) y del programa (con los requisitos de uso: mercado, escuela...) y mediante las leyes de composición (retícula, ejes, simetría...) y combinando un máximo de adecuación con una máxima economía, se elabora la planta del edificio que resuelve los requisitos planteados.

La modernidad arquitectónica abandona la noción del tipo como convención tipológica. Para los teóricos del Movimiento Moderno el tipo se entiende como sinónimo de inmovilidad (Moneo, 2004, p.592). Si en el clasicismo, la forma se asimilaba a un tipo que incorpora la experiencia del tiempo, en la Modernidad se ordena la realidad siguiendo un criterio de forma. La configuración de esta estructura organizativa, libre ya de la coacción de los principios compositivos clasicistas, permite la construcción de la forma con identidad y consistencia propias (Piñón, 2006, p.40). El objetivo de la arquitectura es ordenar una estructura organizativa para atribuirle una identidad. Como señala Rafael Moneo (2004) "el tipo define la lógica interna de las formas" (p.600). Así, la forma se concibe como una manifestación sensitiva de la constitución interna de las cosas.

En el inicio del aprendizaje de Proyectos se plantean unas bases sobre las que trabajar y apoyarse. Se parte de la Teoría del Proyecto de Helio Piñón (2006) con el análisis y el proyecto a partir de obras de arquitectura ejemplares. El análisis de las obras de los maestros permite gradualmente incorporar estrategias y criterios para el proyecto. En las obras de referencia se identifican una serie de relaciones y arquetipos formales que dotan al proyecto de criterios de consistencia: orden, repetición, ritmo, unidad, continuidad y articulación. En el análisis se indaga en la cohesión interna de las partes de una obra arquitectónica desarrolladas de un modo lógico. La simultaneidad del análisis crítico de obras de otros arquitectos, y la adecuación a unas limitaciones funcionales o constructivas propician la profundización en el conocimiento arquitectónico y contribuye tanto a la definición arquitectónica como a enriquecer el bagaje y los recursos del estudiante. Así, se parte de la presencia de ciertas constantes formales que dotan de identidad a la obra y de su transformación y adaptación a las condiciones del proyecto. Como afirma Álvaro Siza "la arquitectura es cada vez más un problema de uso y de referencia a unos modelos. Los arquitectos no inventan nada; trabajan continuamente con modelos 

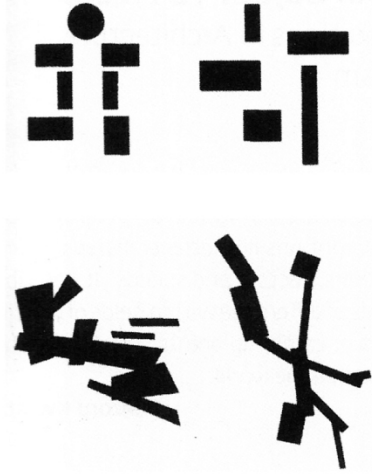

Estrategias organizativas: clásica axial; moderna centrípeta, colisión fragmentos y elementos conectados. Stan Allen

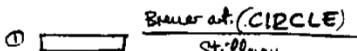

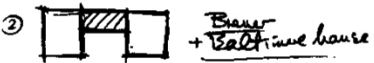

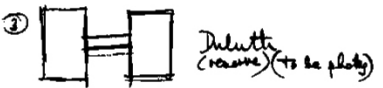

(4)

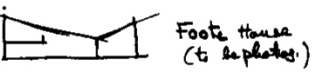

(5)

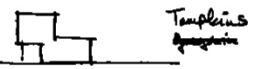

Dibujo clasificatorio de las casas de Marcel Breuer: casa larga y casa binuclear
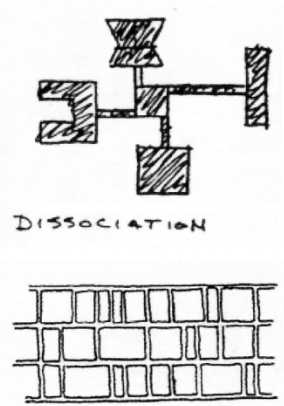

ASSOCIATION

Composición por partes y sistema / trama en tapiz "Mat building". Shadrach Woods.

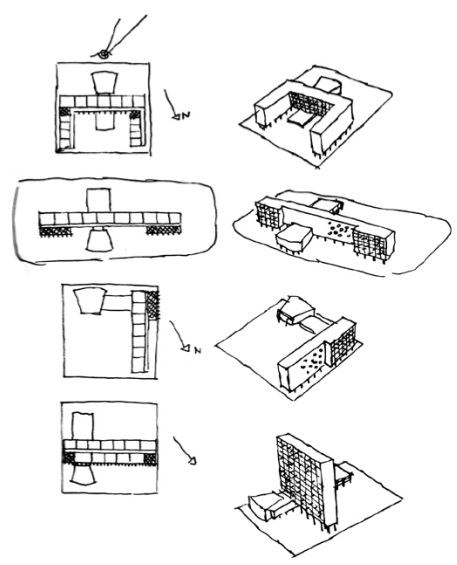

Composición por partes: articulación de alas especializadas. Oscar Niemeyer y Le Corbusier

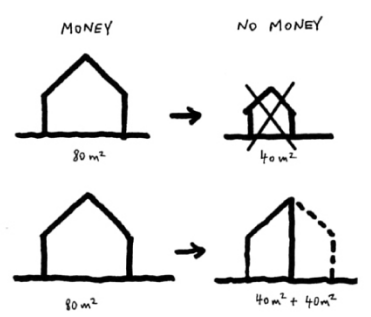

Diseño participativo y habitabilidad global. Construcción en fases. Elemental. Alejandro Aravena.
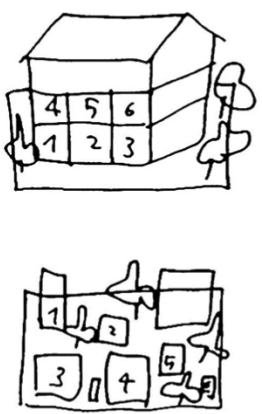

Forma compacta y pabellones. Kazuyo Sejima. Sanaa

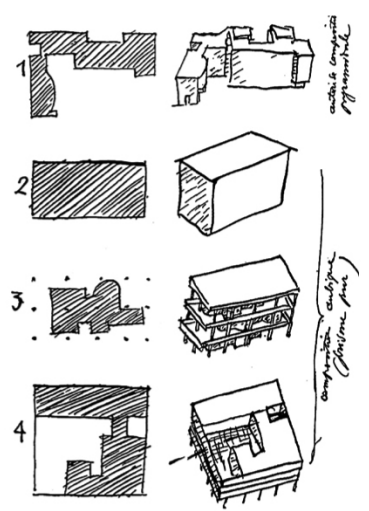

Las cuatro composicones, 1929: La Roche, Garches, Túnez, Villa Savoye. Le Corbusier
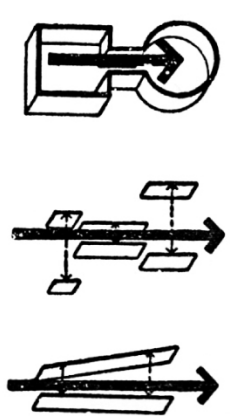

Recorrido y experiencia del espacio a través del recorrido. Contraste entre formas, expansión y dilatación. Michael Leonard

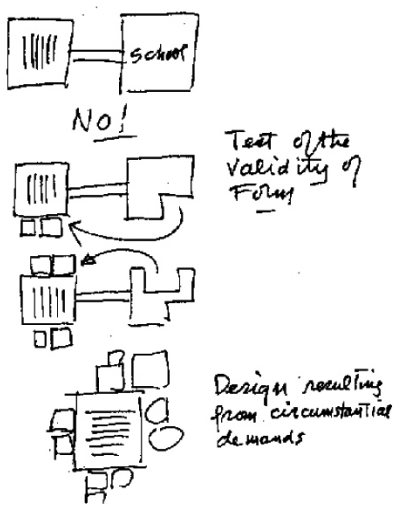

Evolución y análisis del proyecto como resultado de las demandas específicas. Louis Kahn

Figura 2: Tipos. Estrategias organizativas y compositivas. Dibujos de: (1) Stan Allen, (2) Oscar Niemeyer, (3) Le Corbusier, (4) Marcel Breuer, (5) Alejandro Aravena, (6) Michael Leonard, (7) Shadrach Woods, (8) Kazuyo Sejima y (9) Louis Kahn. Fuente: Composición del autor. 
que transforman en respuestas a los problemas con que se enfrentan" (Curtis, 2012, p.594). Se pretende con ello centrar el conocimiento de la arquitectura en lo esencial de la forma, examinando los invariantes formales que se manifiestan en ejemplos diversos de la tradición, analizando los principios permanentes y sus transgresiones.

\subsection{De organizar funciones a ordenar espacios}

Se aborda el vínculo entre el edificio y la utilidad y se propone analizar el grado de adecuación entre el marco físico y las actividades que se desarrollan, analizando los indicios de uso y las relaciones que se establecen, la correspondencia entre los espacios de soporte y las transformaciones introducidas por los usuarios y el grado de flexibilidad. En definitiva, comprender la permanencia y el cambio en arquitectura. La conversación entre Mies van der Rohe y Hugo Häring es reveladora de permanente debate entre la universalidad del proyecto frente a la especificidad del programa: " $\mathrm{iHaz}$ tus espacios lo suficientemente grandes, hombre, de forma que se pueda andar por ellos de forma libre, y no sólo en una dirección predeterminada! O ¿estás totalmente seguro de cómo serán usados?. No sabemos en absoluto si la gente hará con ellos lo que esperamos. Las funciones no son tan claras ni tan constantes: cambian más rápido que el edificio" (Shulze, 2016, p.109). Así, para Mies van der Rohe, la flexibilidad es la característica más importante de sus edificios, no la expresión de la función. La forma es más permanente que el uso que acoja.

A medida que las actividades se manifiestan y fijan una pauta colectiva se establece el requisito de un ámbito que las acoja y propicie su desarrollo. La actividad se formaliza y establece un sistema de relaciones articulado por la arquitectura. Así, la organización del espacio es un reflejo de los modos de vida y es la expresión de una cultura. Para Louis Kahn, la arquitectura parte del estudio de los patrones de las relaciones sociales y el análisis de las necesidades. Para Aldo van Eyck proviene de las raíces culturales en la configuración del hábitat humano.

La arquitectura va más allá de encajar los usos en zonas dimensionadas. Kahn afirma: "creo que el primer acto del arquitecto es tomar el programa que le llega y cambiarlo; no cumplirlo, sino colocarlo en el ámbito de la arquitectura, que es colocarlo en el ámbito de los espacios" (Curtis, 2012, p.520). Así, Louis Kahn define la arquitectura como una creación meditada de espacios.

La resolución arquitectónica debe transcender el diagrama funcional, no es sólo un instrumento para resolver unas funciones. Los ejercicios planteados en este apartado deben dar cabida a las actividades humanas requeridas pero, como escribe Carlos Marti Arís (2014) "a partir de la mera utilidad no puede alcanzarse una formulación arquitectónica" (p.84). Apelando a la capacidad ordenadora del arquitecto, el proyecto arquitectónico debe construir el marco donde se establece el acontecer humano y construir espacios que evoquen una determinada sensación de uso.

\section{Topos}

La noción del lugar constituye otro concepto clave en el desarrollo del proyecto, desde el entendimiento de la arquitectura como vínculo con el sitio. En esta fase de análisis y proyecto, realizada de forma simultánea con las anteriores, se pretende desarrollar el proyecto desde la concienciación medioambiental y abordar la relación de la arquitectura con su medio físico entendiéndola como superposición y contribución a lo existente, no como imposición en lo preexistente. Así, las preexistencias de un sitio son el mayor soporte de la imaginación.

Se trata de identificar las características del medio físico existente y evaluar su incidencia en el proyecto: la forma del emplazamiento, la topografía y la implantación, la influencia del clima y la orientación, el carácter del medio donde se sitúa, el paisaje o las vistas. Mediante el dibujo analítico del emplazamiento y una mirada atenta al entorno se establecen intensos vínculos con el lugar. María Isabel Alba Dorado (2013) reivindica la importancia del dibujo analítico del sitio "el dibujo necesita, pues, de la acción de una mirada atenta, una mirada que vigila y explica, que observa y dibuja y que procura ese conocimiento que permite extraer del contexto aquellos datos necesarios que más tarde entrarán a formar parte del proyecto" (p.199).

El proyecto establece un sistema de relaciones con e sitio para vincularlo con el medio del territorio de la naturaleza o de la ciudad. Estos vínculos con el sitio, que Louis Kahn denomina "arquitecturas de conexión", son los espacios intermedios de la plataforma, el antepatio, el patio, el porche, la terraza... y establecen un sistema que proporciona a través de los itinerarios y los recorridos una liturgia del acceso al proyecto. En definitiva, se trata de estudiar en este apartado cómo el sitio transforma el proyecto y cómo el edificio revela y activa los atributos del sitio (Figura 3)

El objetivo de los ejercicios propuestos en este apartado es entender la arquitectura como material que construye la ciudad y la naturaleza de las relaciones que el proyecto establece con el sitio. Lo importante no es tanto la forma de los edificios, como las formas entre los edificios: el espacio urbano (Allen, 2009, p.218). La lectura paralela del libro titulado La humanización del espacio urbano. La vida social entre los edificios de Jan Gehl (2008) y los capítulos dedicados a la vida entre los edificios, orientarán el proyecto del espacio libre público tratando de agrupar e integrar las actividades planteadas, atraer a todas las personas (accesibilidad universal), abrir para fomentar un enriquecedor intercambio de experiencias y propiciar las secuencias espaciales en los recorridos, los lugares de estancia y las actividades (educativas, deportivas, culturales, lúdicas o recreativas). Asimismo, se plantea desarrollar el proyecto desde la concienciación medioambiental, con la renaturalización de la ciudad que contribuya a la reducción de la contaminación y del tráfico, y fomentando los recorridos a pie; en definitiva, reivindicando una ciudad saludable y viva, con una mayor dotación de espacios públicos de uso ciudadano. 


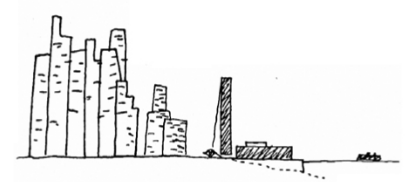

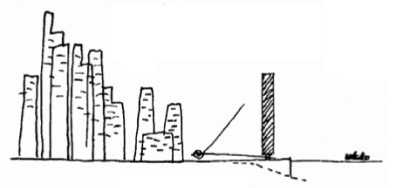

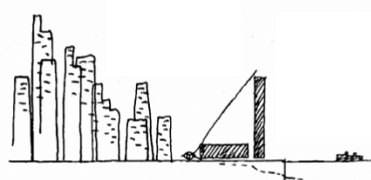

Análisis del emplazamiento y valoración de la implantación del ediicio en el sitio. Oscar Niemeyer

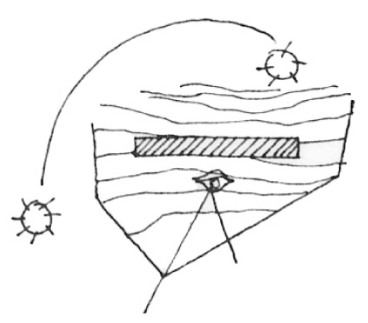

Análisis del sitio: topografía, orientación, soleamiento y vistas. Oscar Niemeyer
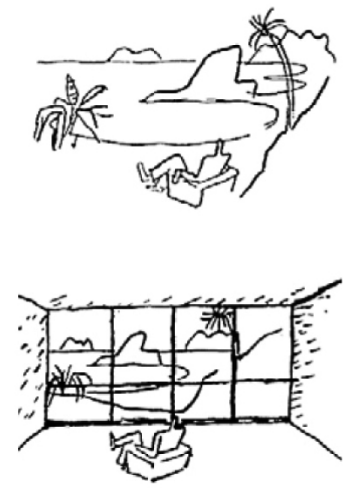

Enmarcar el paisaje. Le Corbusier

Orientación, paisaje, vistas desde el emplazamiento. Le Corbusier
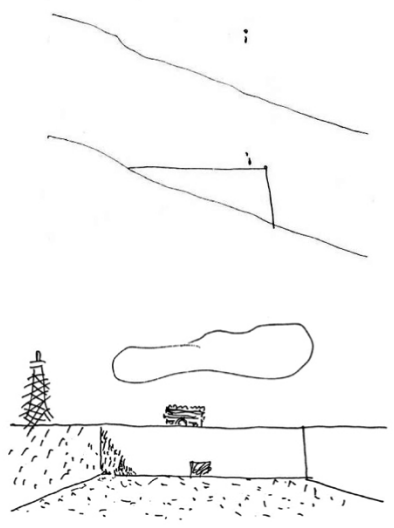

Plataforma y recinto: dos formas complementarias de fundar un lugar. Sverre Fehn y Le Corbusier
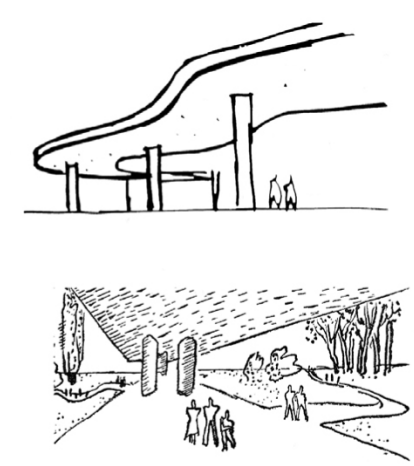

Porche: espacio cubierto y abierto. Arquitectura y clima: sistemas pasivos. Oscar Niemeyer y Le Corbusier
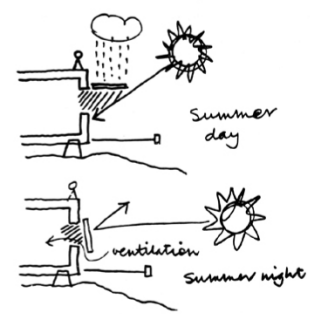

Ralph Erskine
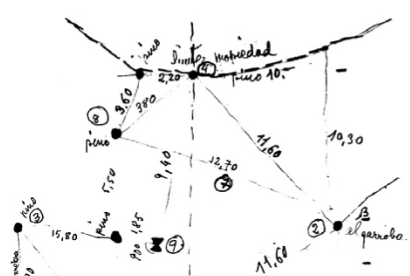

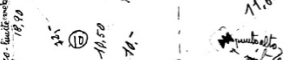

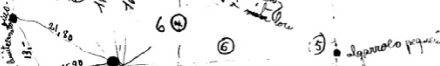

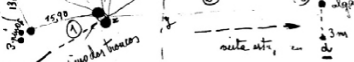

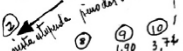

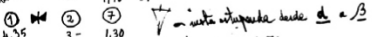

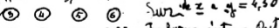

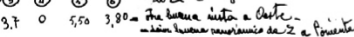

Análisis del sitio y de las preexistencias de la parcela, vegetación y vistas. José Antonio Coderch
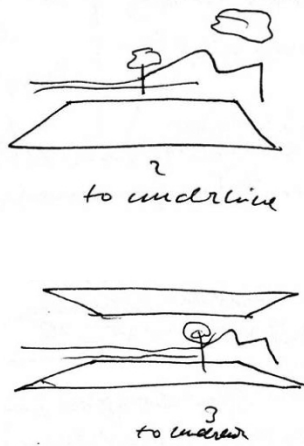

Subrayar un paisaje y enmarcar un paisaje. Plataforma y porche. Alberto Campo Baeza

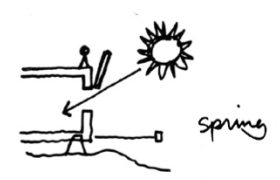

Figura 3: Topos. La arquitectura como vínculo con el sitio. Dibujos de: (1,2) Oscar Niemeyer, (3) José Antonio Coderch, $(4,5)$ Le Corbusier, (6) Alberto Campo Baeza, (7) Sverre Fehn, Le Corbusier, (8) Oscar Niemeyer, Le Corbusier y (9) Ralph Erskine.

Fuente: Composición del autor. 
En un segundo nivel de análisis se presenta el proyecto como dispositivo de control ambiental capaz de proporcionar las condiciones de confort a los usuarios mediante el uso de estrategias y dispositivos de contro climático. Se trata de identificar la relación del edificio con el medio existente y evaluar la sensibilidad al entorno a través de las denominadas "reacciones poéticas" de Le Corbusier.

Alberti reformuló el concepto de "utilitas" en "commoditas", introduciendo la noción de confort. En relación con el cambio climático y la sostenibilidad energética que demanda la reducción de energía en el sector de la edificación, se introducen los conceptos de sostenibilidad, adecuación climática y eficiencia energética. Se trata de adaptar el proyecto al sitio, a las condiciones específicas del clima y del territorio, en relación tanto a los umbrales de habitabilidad y confort demandados como por las distintas escalas de la implantación del proyecto. Desde las "arquitecturas de conexión", de Louis Kahn, al reto ecológico de Ralph Erskine o a la sensibilidad medioambiental del "Form follows energy", de Charles Correa (Correa, 2008, p.19).

Así, la obra de arquitectura es capaz de reconocer y expresar los atributos del sitio mediante el entendimiento del "genius loci", que denomina Christian Norberg Shulz (Norberg-Schulz, 1986, p.10). Para Le Corbusier, "la arquitectura expresa el sitio" (2008, pp.29-30). E respeto por el paisaje y el entorno de las preexistencias ambientales y la voluntad de continuidad contribuye con sentido pedagógico a la concienciación medioambienta en una arquitectura modélica en su comprometida relación con el medio natural y la sostenibilidad, reivindicando lo que Josep Maria Montaner denomina como la belleza de las arquitecturas ecológicas (Montaner, 2011, p.159).

\section{Tectónica}

La construcción y la estructura constituyen la base de la expresión arquitectónica, a partir de la lógica inherente de cada material y de las reglas sintácticas de los sistemas constructivos (Figura 4). Como afirma Auguste Perret, "la construcción es la lengua madre del arquitecto. El arquitecto es un poeta que piensa y habla en términos constructivos" (Muñoz Cosme, 2008, p.146). La construcción y la estructura constituyen la base de la expresión arquitectónica, a partir de la lógica inherente de cada material y de las reglas sintácticas de la estructura. Jørn Utzon, en el artículo titulado La importancia de los arquitectos, escribe: "Si uno quiere llegar a ser arquitecto, habrá de dominar la tecnología para poder desarrollar sus ideas, para demostrar el acierto de sus intenciones, para construir sus sueños" (Utzon, 1995, p.12). Así, la técnica constituye la mediación entre la idea, la decisión intelectual y la realidad construida.

El maestro noruego Sverre Fehn afirma que la idea poética necesita un soporte, que es la construcción, para existir. Por tanto, la condición esencial de la concepción arquitectónica es la técnica. Como afirma el profesor
Helio Piñón (2006): “la construcción es un instrumento para concebir, no una técnica para resolver" (p.122). No hay proyecto arquitectónico sin conciencia constructiva. Proyectar es construir, y en consecuencia, ordenar y relacionar elementos materiales para generar sistemas espaciales caracterizados por la consistencia formal. La tectónica es la condición de la forma arquitectónica que dota de un orden al material. La construcción impone una disciplina al proyectar.

El rigor que impone el sistema constructivo no supone una restricción formal, sino que es un instrumento de generación poética, de construcción y no de constricción, de limitación. Las leyes que impone el proceso constructivo se manifiestan como una necesidad para la arquitectura. El modo de trabar, ensamblar, unir y ordenar materiales y espacios estructura el proyecto.

Esta reflexión sobre la técnica examina también los planteamientos teóricos del alemán Gottfried Semper, que divide la forma construida en dos procedimientos materiales: la tectónica de la trama, en la que las distintas partes se conjugan constituyendo una única unidad espacial, y la estereotómica, de la masa que trabaja a compresión (Semper, 2014, p.295). La arquitectura estereotómica es la arquitectura del podio, del basamento, del estilobato. Es un sistema estructural continuo y configura una arquitectura masiva, pesada y anclada al suelo mediante una gran continuidad constructiva. La arquitectura tectónica es un sistema estructural donde la construcción es articulada. Es un sistema estructural articulado y configura una arquitectura ligera.

Viollet-le-Duc explica la construcción como la resistencia de los materiales a la acción gravitatoria. La estructura asciende desde el suelo. Los cimientos construyen la lógica de la planta y la gravedad organiza el trabajo de los materiales organizados en sistemas. A este movimiento ascendente de la construcción se refiere Louis Kahn: "Si nos acostumbrásemos a dibujar como construimos, de abajo arriba, deteniendo nuestros lápices en las juntas del vertido (del hormigón) o de la fábrica, el ornamento derivaría de nuestro amor por la perfección de la construcción y elaboraríamos nuevos métodos constructivos" (Sabini, 1994, p.114).

El análisis que se propone realizar comprende la reconstrucción de los sistemas constructivos del proyecto. Se recurre a la construcción de una maqueta que ilustre el conocido dibujo de Albert Frey titulado "los elementos de la casa", donde dibuja la progresión, desde el sitio y la plataforma a la construcción de la estructura resistente, el muro de carga o la estructura de pilares, a la ejecución del cerramiento del ambiente y finalmente la construcción de la cubierta que se apoya en la estructura. Este movimiento hacia arriba permite explicar el proceso constructivo y reivindica la denominada "arquitectura física" de Alejandro de la Sota, donde se reconocen los elementos que componen el proyecto (Sota, 2002, p.171).

La maqueta de la secuencia constructiva señala también otra dimensión analítica en el uso de la maqueta como instrumento pedagógico complementario a la maqueta 


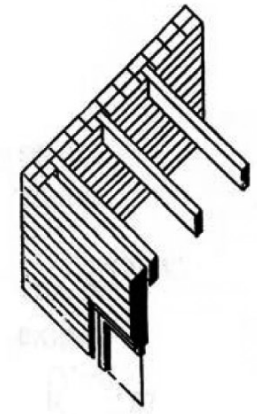

Sistemas estructural de crujías de muros de carga y viguetas. Coincidencia entre soporte, cerramiento y compartimentación

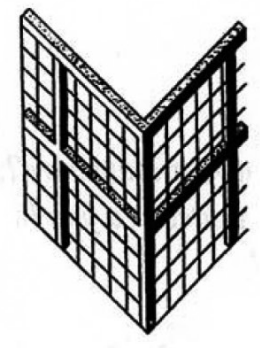

Sistema estructural de entramado como estructura portante formado por pilares y vigas. Theo van Doesburg. Sistemas constructivos 1923

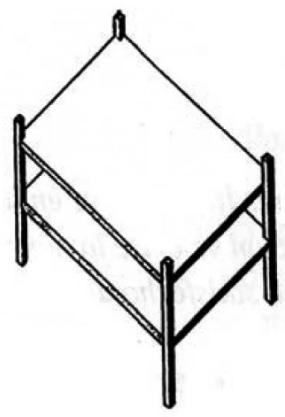

Sistema estructural de losas rígidas sobre pilares. La estructura portante constituye una estructura espacial

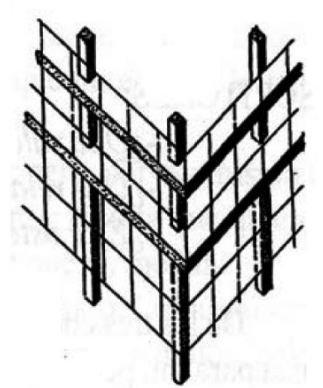

Autonomía de la estructura y el cerramiento. El voladizo como caracterización formal moderna

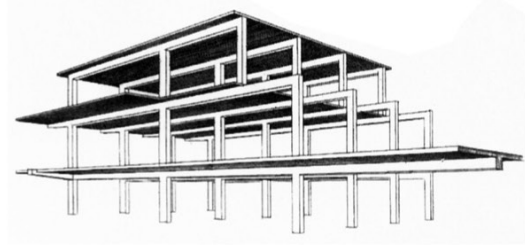

Estructura de pilares y jácenas: pórticos. La estructura portante contribuye a estructurar el espacio. Mart Stam

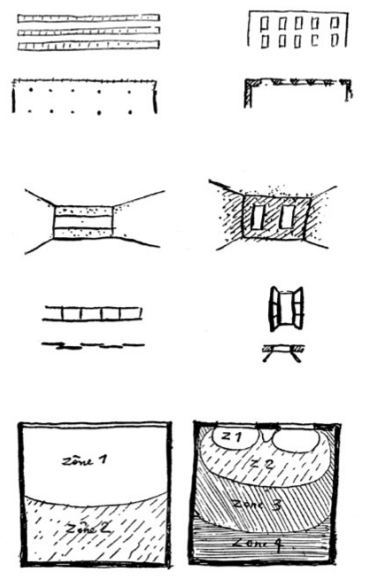

1. Los pilotis 2. La cubierta jardín, 3. Planta libre, 4. Ventana corrida, 5. La fachada libre

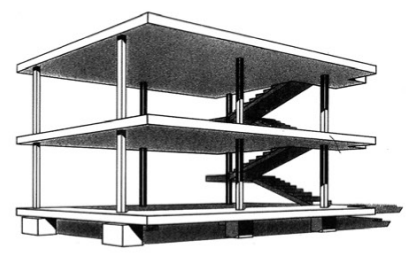

Sistema dominó de Le Corbusier: losas rigidas voladas sobre pilares

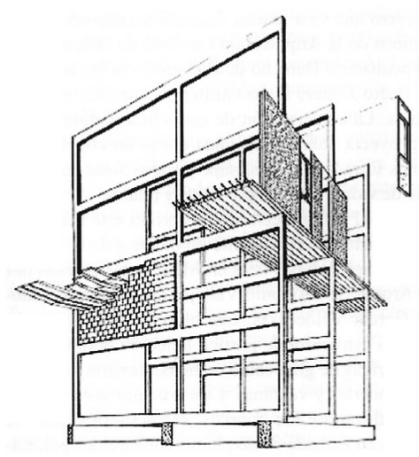

Los cinco puntos de una nueva arquitectura. Le Corbusier

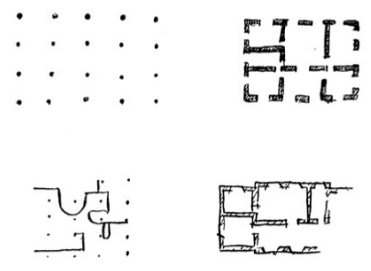

Sosen

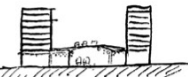

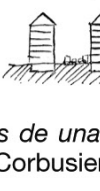
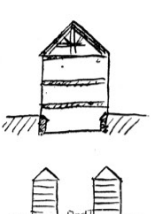

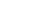

Figura 4: Tectónica. Sistemas constructivos y espaciales. Dibujos de: (1,2,3,4) Theo van Doesburg, (5) Mart Stam, $(6,7,8)$ Le Corbusier y (9) Francisco Javier Sáenz de Oíza.

Fuente: Composición del autor. 
del vacío ensayado al inicio del curso. Simultáneamente a la ejecución de la maqueta, el dibujo de la sección del proyecto constituye la verificación del proyecto. La interdependencia entre el sistema constructivo y el sistema espacial es el atributo que contribuye a la coherencia constructiva del orden estructural. En cuanto al análisis de los materiales utilizados se puede apuntar, como afirma Mies van der Rohe, que en realidad no esperamos nada de los materiales, sino únicamente de su empleo correcto: "un material sólo vale lo que hagamos con él" (Neumeyer, 2000, p.13)

En la primera parte del ejercicio se desarrolla el análisis de la estructura resistente y del sistema constructivo del proyecto. Se identifica la estructura y los sistemas constructivos (cerramientos, compartimentación, cubiertas, instalaciones) y a través de la maqueta se presenta la secuencia constructiva del proyecto. El análisis profundiza en el papel que desempeña la estructura en la configuración final del proyecto y en los materiales. En la arquitectura tradicional un único material cumple diversas funciones, como un ladrillo que es, a la vez, estructura, cerramiento o compartimentación. En la construcción moderna, se produce la especialización de los elementos constructivos que responden específicamente a una función (estructural, aislamiento, impermeabilización...).

Si en el anterior epígrafe se analizaba la incidencia del sitio en la arquitectura, en este ejercicio se plantea examinar la incidencia de la estructura en la configuración del proyecto. Para Mies van der Rohe (2015), "la visualización del espacio debe estar apoyada en el conocimiento estructural. Diseño sin conocimiento estructural resultará diletantismo. Por esta razón hacemos de la construcción un requisito previo a la planificación arquitectónica" (p.8). Se trata de analizar el sistema constructivo espacial de un ejemplo proporcionado y alterarlo introduciendo otro sistema constructivo como elemento resistente. Se trata de analizar y descubrir el orden y los principios que rigen la construcción. Louis Kahn afirma que la forma se origina en un sistema constructivo. Por tanto, el objetivo es obtener otro proyecto distinto, conservando al máximo la configuración del modelo en todos sus aspectos (organización, dimensiones, espacialidad...) y modificando todo aquello a lo que obligue la lógica del nuevo sistema adoptado. Así se puede comprender y comparar la distinta incidencia de los sistemas constructivos en la configuración del espacio: de la estructura resistente reticular que libera la planta y permite la autonomía de la compartimentación y la estructura con la presencia de los voladizos como caracterización formal moderna, al sistema de crujías de muros de carga, cuyas trazas determinan la compartimentación y la espacialidad.

\section{Conclusión}

Esta aproximación a la docencia para un curso inicia de proyectos reivindica el conocimiento, el sentido y la utilidad de lo que se hace. La arquitectura no es sólo un "hacer" sino un "saber hacer", como afirma el profesor Carlos Martí Arís (2005): "tras toda gran obra existe un esfuerzo reflexivo, un pensamiento teórico activo" (p.11). El programa planteado trata de aproximar al estudiante, en su acercamiento al conocimiento arquitectónico, tanto a la obra arquitectónica como a la construcción teórica que la sustenta. A través de la conceptualización de los fundamentos, el curso prepara a los arquitectos para el pensamiento, la reflexión y a adoptar una posición intelectual y teórica sobre la práctica arquitectónica, asimilando un contenido relevante para el proceso de proyecto y fomentando una enseñanza formativa, tanto en el conocimiento disciplinar como el pensamiento crítico que propicie, con la guía docente, el entendimiento del proyecto como producto cultural.

Tanto la localización y la situación del proyecto en las condiciones específicas de un sitio (Topos), de las condiciones del programa (Tipos) o de las soluciones estructurales (Tectónica) sitúan simultáneamente al estudiante ante los factores con los que trata la arquitectura y que inciden en la definición de la forma arquitectónica y en los espacios en los que se desarrollan las actividades humanas. Los ejercicios propuestos tratan de profundizar en los temas que debe abordar el proyecto de arquitectura en su relación con el sitio, en la definición de ámbitos urbanos y públicos (Topos), y en la lógica interna de la elaboración del proyecto atendiendo simultáneamente a los aspectos de composición y ordenación espacial (Tipos) o a la relación con los sistemas constructivos y espaciales y la atención a los materiales (Tectónica). Los ejercicios planteados tratan de aproximarse a la complejidad del hecho arquitectónico y propician el desarrollo de actitudes, habilidades y capacidades en la investigación proyectual. Se enmarcan en los actuales retos ecológicos y económicos (Reducción, Re-uso, Reciclaje) y reivindican la vocación de servicio de los arquitectos, capaces de contribuir desde la disciplina a mejorar la calidad de vida de las personas.

El adecuado conocimiento de un curso introductorio que plantea las bases de la práctica del proyecto se logra con un conjunto transmisible de saberes que provienen de la cultura de la propia disciplina arquitectónica y que derivan de las obras arquitectónicas. Como señala Rafael Moneo (2017), "acceder a la arquitectura, y si se quiere ser más preciso, a la práctica de la arquitectura desde el conocimiento, me sigue pareciendo el mejor camino para la formación del arquitecto" (p.16). Así, se propone esta aproximación al entendimiento en términos espaciales, formales y constructivos de cómo se produce la arquitectura, reivindicando el conocimiento como la base de la enseñanza de la arquitectura.

\section{Agradecimientos}

Se agradece al Departamento de Proyectos Arquitectónicos de la Escuela Técnica Superior de Arquitectura de Barcelona (ETSAB), Universitat Politècnica de Catalunya (UPC). 
Cómo citar este artículo/How to cite this article: Ferrer, J. (2019). Primera piedra. Bases para el Proyecto Arquitectónico. Estoa, Revista de la Facultad de Arquitectura y Urbanismo de la Universidad de Cuenca, 8(15), 45-55. doi:10.18537/est.v008.n015. a04

\section{Bibliografía}

Allen, S. (2009). Practice: Architecture, Technique + Representation. Londres, UK: Routledge.

Alba Dorado, M. I. (2013). Manos que piensan. Reflexiones acerca del proceso creativo del proyecto de arquitectura. EGA Expresión Gráfica Arquitectónica, (22), pp. 196-203. doi: 10.4995/ ega.2013.1694

- Alba Dorado, M. I. (2016). La mirada atenta. Aproximaciones a la creación arquitectónica. EGA Expresión Gráfica Arquitectónica, (27), pp. 88-95. doi: 10.4995/ega.2016.4732

- Capitel, A. (2016). Tres sistemas arquitectónicos. Patios, partes y forma compacta. Barcelona, España: Fundación Arquia. Los libros de la Catarata.

- Correa, C. (2008). Un lugar a la sombra. Barcelona, España: Fundación Caja de Arquitectos.

- Cortés, J. A. y Moneo, R. (1976). Comentarios sobre dibujos de 20 arquitectos actuales. Barcelona, España: ETSAB.

- Curtis, W. J. R. (2012). La arquitectura moderna desde 1900. Londres, UK : Phaidon.

- Frampton, K. (1999). Estudios sobre la cultura tectónica. Poéticas de la construcción en la arquitectura de los Siglos XIX y XX. Madrid, España: Akal.

- García del Monte, J. M. (2017). Guía para estudiantes de arquitectura. Consejos para el proyecto. Madrid, España: Fundación Arquia. Los libros de la Catarata.

- Gehl, J. (2008). La humanización del espacio urbano. La vida social entre los edificios. Barcelona, España: Editorial Reverté.

- Labarta, C. (2011). Aptitud y actitud: parámetros integrados para una metodología docente. En. C. Labarta, I. Bergera (Eds.), Metodología docente del proyecto arquitectónico (34-45). Zaragoza, España: Prensas Universitarias Universidad de Zaragoza.

- Le Corbusier (2008). Mensaje a los estudiantes de arquitectura. Buenos Aires, Argentina: Infinito.

- Martí Arís, C. (2014). Las variaciones de la identidad. Ensayo sobre el tipo en arquitectura. Barcelona, España: Fundación Arquia.

- Martí Arís, C. (2005). La Cimbra y el arco. Barcelona, España: Fundación Arquia.

- Mies van der Rohe, L. (2015). Sobre la enseñanza de la arquitectura y los valores. A\&P Continuidad, (2), pp. 8-11.
- Moneo, R. (2004). Sobre la noción del tipo. En Rafael Moneo 1967-2004 [imperative anthology antología de urgencia] (pp. 584-606). Madrid, España: El Croquis.

Moneo, R. (2017). Rafael Moneo: una manera de enseñar arquitectura. Lecciones desde Barcelona, 1971-1976. Barcelona, España: Edicions UPC.

Montaner, J. M. (2011). La modernidad superada. Ensayos sobre arquitectura contemporánea. Barcelona, España: Editorial Gustavo Gili.

Norberg-Schulz, C. (1986). Genius loci. Paesaggio, ambiente, architettura. Milán, Italia: Electa.

Muñoz Cosme, A. (2008). El proyecto de arquitectura. Barcelona, España: Reverté.

Neumeyer, F. (2000). Mies van der Rohe. La palabra sin artificio. Reflexiones sobre arquitectura 1922-1968. El Escorial, España: El Croquis Editorial.

Piñón, H. (2006). Teoría del proyecto. Barcelona, España: Edicions UPC.

- Sabini, M. (1994). Louis I. Kahn. Barcelona, España: Ediciones del Serbal.

- Scott, G. (1970). La arquitectura del humanismo: un estudio sobre la historia del gusto. Barcelona, España: Barral.

- Schulze, F. y Windhorst, E. (2016). Ludwig Mies van der Rohe: una biografía crítica. Barcelona, España: Editorial Reverté.

- Semper, G. (2014). Escritos fundamentales de Gottfried Semper. El fuego y su protección. Edición y prólogo de Antonio Armesto. Barcelona, España: Fundación Arquia.

- Sota, A. (2002). Alejandro de la Sota. Escritos, conversaciones, conferencias. Barcelona, España: Editorial Gustavo Gili.

- Utzon, J. (1995). La importancia de los arquitectos. En Utzon, J. (Ed.), Jørn Utzon (pp. 12-13). Madrid, España: Ministerio de obras Públicas, Transportes y Medio Ambiente.

- Vacchini, L. (2009). Obras maestras. Barcelona, España: Gustavo Gili.

- Vallespín, A., Cervero N., y Cabodevilla-Artieda, I. (2017). Los collages de la casa Resor de Mies van der Rohe como transparencia fenomenal. EGA Expresión Gráfica Arquitectónica, (31), 140-149. doi: 10.4995/ega.2017.7392

- Vitruvio (1980). Los diez libros de arquitectura. Tr. y notas de Blázquez, A. Madrid, España: Iberia. Zevi, B. (1991). Saber ver la arquitectura. Barcelona, España: Poseidón. 